\section{Prevalência de depressão \\ e fatores associados em \\ comunidade de baixa renda de \\ Porto Alegre, Rio Grande do Sul}

\section{Prevalence of depression and associated factors in a low income community of Porto Alegre, Rio Grande do Sul}

\author{
Ricardo Vivian da Cunha' \\ Gisele Alsina Nader Bastos" \\ Giovâni Firpo Del Duca"' \\ 'Programa de Pós Graduação em Saúde da Família, Hospital Moinhos de Vento, \\ Porto Alegre, RS. \\ "Escola de Gestão em Saúde, Hospital Moinhos de Vento, Porto Alegre, RS \\ e Departamento de Saúde Coletiva da Universidade Federal de Ciências da \\ Saúde de Porto Alegre, RS. \\ "' Universidade Federal de Santa Catarina, Florianópolis, SC.
}

\section{Resumo}

Objetivo: Estimar a prevalência e os fatores demográficos e socioeconômicos associados à depressão em adultos e idosos em uma comunidade de baixa renda de Porto Alegre, Rio Grande do Sul. Métodos: Estudo transversal realizado com adultos com $\geq 20$ anos de idade residentes nos distritos sanitários da Restinga e Extremo Sul, na cidade de Porto Alegre, Rio Grande do Sul, de julho a dezembro de 2009. A variável dependente do estudo foi a depressão, avaliada pela Escala de Depressão Pós-Natal de Edimburgo. As variáveis independentes foram sexo, idade, situação conjugal atual, escolaridade e nível econômico. Empregou-se teste qui-quadrado de Pearson na análise bruta e regressão de Poisson com variância robusta na análise ajustada. Resultados: Dentre os entrevistados, a prevalência de depressão encontrada foi de 16,1\% (IC95\%: 14,9\%; 17,4\%). Após análise ajustada, observou-se que a depressão esteve associada ao sexo feminino ( $\mathrm{RP}=2,38$ ). Além disso, observou-se tendência de maiores ocorrência de depressão conforme o aumento da faixa etária e diminuição dos níveis de escolaridade e renda. Conclusões: Os valores de depressão encontrados foram semelhantes a outros estudos populacionais. Atenção específica deve ser destinada a mulheres e indivíduos de baixa escolaridade, que apresentaram maiores ocorrências de depressão.

Palavras-chave: Depressão. Estudos transversais. Prevalência.

Fonte de financiamento: Pesquisa realizada no âmbito do Projeto Desenvolvimento de Técnicas de Operação e Gestão de serviços de Saúde em uma Região Intramunicipal de Porto Alegre - Distritos da Restinga e Extremo-Sul, de acordo com o Programa de Apoio ao Desenvolvimento Institucional do Sistema Único de Saúde (PROADI-SUS), firmado entre o Ministério da Saúde e a Associação Hospitalar Moinhos de Vento, por meio do termo de ajuste de número 06/2008, assinado em 17 de novembro de 2008.

Correspondência: Ricardo Vivian da Cunha. Programa de Pós Graduação em Saúde da Família Hospital Moinhos de Vento, Porto Alegre, RS. Rua São Francisco, 859/203 - Santana - Porto Alegre, RS CEP 90620-070. E-mail: rivcpsi@yahoo.com.br 


\section{Abstract}

Objective: To estimate the prevalence and demographic and socioeconomic factors associated with depression in adults and in the elderly in a low income community of Porto Alegre, Rio Grande do Sul. Methods: Cross-sectional study of adults with $\geq 20$ years of age living in the Health Districts of Restinga/Extremo Sul, Porto Alegre, Rio Grande do Sul, between July and December 2009. The dependent variable was depression measured by the Edinburgh Postnatal Depression Scale. Independent variables were sex, age, current marital status, educational level and economic level. The Chisquare test was used for the crude analysis and Poisson regression with robust variance for the adjusted analysis. Results: Among respondents, the prevalence of depression was $16.1 \%$ (95\% CI: $14.9 \%, 17.4 \%)$. After adjusted analysis, we found that depression was associated with the female gender $(\mathrm{PR}=2.38)$. In addition, there was a trend of higher occurrence of depression with increasing age and decreasing levels of schooling and income. Conclusions: The values of the results for depression were similar to other population studies. Specific attention should be given to women and individuals with low schooling.

Keywords: Depression. Cross-sectional. Prevalence.

\section{Introdução}

A depressão representa um importante e crescente problema para a saúde pública. Acredita-se que seja a principal causa de incapacidade mental em termos mundiais e estima-se que, até 2020 , seja a segunda causa de incapacidade para a saúde ${ }^{1,2}$.

Em sua origem, a depressão é fruto de fatores genéticos, bioquímicos, psicológicos e sociofamiliares, sendo estudada sob diferentes abordagens. É classificada como um conjunto de transtornos, que se manifestam numa certa duração, frequência e intensidade, que os manuais psiquiátricos mundialmente reconhecidos e atualmente em vigor descrevem minuciosamente. É estudada como diagnóstico, sendo sistematizada pelo Manual Diagnóstico e Estatístico de Transtornos Mentais (DSM-IV), no item "Transtornos do Humor" e "Transtornos Afetivos", pela Classificação internacional de doenças e problemas relacionados à saúde (CID-10) $)^{3,4}$.

No CID-10, a depressão encontra-se classificada nos itens F32-F33 (classificada em três graus: leve, moderado ou grave), no qual o paciente apresenta um rebaixamento do humor, redução da energia e diminuição da atividade ${ }^{4}$. Pessoas que sofrem de depressão experimentam sintomas como sentimentos de tristeza profunda, falta de confiança, visões sobre si e sobre os outros, negativas e, em longo prazo, perda de interesse em atividades, distúrbios de sono e apetite, acompanhados de dores de cabeça e fadiga ${ }^{5}$.

Segundo a Pesquisa Nacional por Amostra de Domicílios divulgada pelo Instituto Brasileiro de Geografia e Estatística, a depressão, identificada por profissional de saúde, atinge 7,8 milhões de brasileiros, o que corresponde a $4,1 \%$ da população ${ }^{6}$. Além disso, a depressão está associada a certas características sociais, como a baixa escolaridade, o desemprego e o baixo nível econômico, de acordo com o sistema de classificação da Associação Brasileira de Institutos de Pesquisa de Mercado ${ }^{7-9}$.

A prevalência na população geral para transtornos depressivos tem alcançado 
números entre $4 \%$ e $10 \%$, sendo observada uma maior incidência em mulheres, variando de $10 \%$ a $25 \%$, enquanto nos homens a porcentagem é de $5 \%$ a $12 \%{ }^{10}$. Outro dado importante é que uma a cada 20 pessoas é atingida por um episódio depressivo durante o curso da vida, e em cada 50 casos diagnosticados com a patologia, um necessita de internação, e $15 \%$ dos deprimidos graves cometem suicídio ${ }^{11}$. O estudo proporcionará subsídios técnicos e científicos para futuras intervenções, visto a carência de outros estudos com o instrumento (EPDS) e com as especificidades da população investigada. Sendo assim, este trabalho busca estimar a prevalência, bem como os fatores demográficos e socioeconômicos associados à depressão em adultos e idosos em uma região economicamente desfavorecida de Porto Alegre, Rio Grande do Sul.

\section{Métodos}

Os dados do presente estudo são originários de amplo inquérito epidemiológico que teve por intuito descrever as condições de saúde da população residente nos Distritos Sanitários da Restinga e Extremo Sul, na cidade de Porto Alegre, Rio Grande do Sul, com a finalidade de planejamento de uma rede de serviços de saúde a ser fixada no local. Os distritos sanitários são formados por 27 vilas, onde vivem cerca de cem mil pessoas. O local foi escolhido em virtude das características de isolamento urbano, elevada densidade populacional, carência de estruturas de atenção à saúde e indicadores sociais que evidenciam situação de vulnerabilidade e risco social.

O cálculo de tamanho amostral utilizou os seguintes parâmetros e estimativas: prevalência de depressão de $13 \%$, intervalo confiança de $95 \%$ e erro aceitável de dois pontos percentuais, com adicional de $10 \%$ para perdas e recusas, resultando em 1.184 sujeitos. No entanto, outros cálculos de amostra também foram realizados e, em função dos diversos desfechos estudados, o maior tamanho amostral prevaleceu ( $\mathrm{n}=$ 3.000 sujeitos).
O processo de amostragem por conglomerados em dois estágios teve os 117 setores censitários domiciliares definidos com unidades amostrais primárias e os 29.929 domicílios habitados como unidades amostrais secundárias. A partir de pulos sistemáticos, fizeram parte da amostra todos os indivíduos com idade igual ou superior a 20 anos residentes nos domicílios sorteados. Perdas e recusas foram definidas após a não realização da entrevista em, no mínimo, três visitas efetuadas em dias e horários distintos.

A coleta de dados, realizada de julho a dezembro de 2009, foi conduzida por entrevistadores submetidos a treinamento de 80 horas para a devida aplicação do questionário. A variável dependente do estudo foi o diagnóstico de depressão, avaliada a partir da aplicação da Escala de Depressão Pós-Natal de Edimburgo, que consiste em um instrumento de autoavaliação composto por 10 itens, sendo uma escala curta, de rápida e fácil aplicação, referentes aos sintomas depressivos frequentemente observados. A Escala de Depressão Pós-Natal de Edimburgo (EPDS) é também conhecida como Escala de Depressão de Edimburgo $(E D S)^{12}$. Estudos sugerem que na escala seja suprimido o termo pós-natal, por não ser utilizada somente em situações pós-natal, sendo aplicada em diversos estudos com a população em geral ${ }^{12-17}$.

Apesar do nome, foi escolhido por ter sido utilizado em outros estudos, com população de características sociais semelhantes às de onde ocorreu o atual estudo ${ }^{18-20}$. O referido instrumento foi validadado para comunidade em geral, não sendo exclusivo para os grupos específicos e pode ser utilizado para rastreamento e diagnóstico de depressão em nível populacional, para ambos os sexos ${ }^{19-23}$. Foram definidas como depressão as situações em que os respondentes tiveram escore $\geq 13$, sendo este ponto de corte o padrão ouro ${ }^{20}$.

As variáveis independentes do estudo foram categorizadas do seguinte modo: sexo (masculino e feminino), idade em anos completos (20-29, 30-39, 40-49, 
50-59, 60-69, 70-79 e $\geq 80$ ) escolaridade em anos completos de estudo (0, 1-4, 5-8, $9-11, \geq 12)$, situação conjugal atual - com companheiro(a), solteiro(a) e separado(a)/ viúvo(a) - e nível econômico sendo agrupado em quartis, a partir da aplicação do questionário padronizado da Associação Brasileira de Empresas de Pesquisa ${ }^{24}$.

Na estatística descritiva foram empregadas proporções e respectivos intervalos de 95\% de confiança para variáveis categóricas, assim como médias, amplitudes de variação e desvios-padrão (DP) para variáveis numéricas. Na análise bruta empregou-se o teste qui-quadrado de Pearson para variáveis categóricas dicotômicas e politômicas nominais e tendência linear para variáveis categóricas ordinais. $\mathrm{Na}$ análise ajustada foi empregada a regressão de Poisson com variância robusta, com os resultados expressos em razões de prevalências ${ }^{25}$. Levou-se em consideração a amostragem por conglomerados, bem como a hierarquia entre os possíveis fatores associados com o desfecho para a análise dos dados: no primeiro nível de análise foram incluídas as variáveis demográficas (sexo, idade e situação conjugal) e, no segundo nível, as variáveis socioeconômicas (escolaridade $\mathrm{e}$ nível econômico). Na modelagem estatística, adotou-se a estratégia de seleção "para trás" e um nível crítico de valor $\mathrm{p} \leq 0,20$ para permanência no modelo para controle de confusão, considerando-se estatisticamente significativos valores $\mathrm{p} \leq 0,05$.

O protocolo de pesquisa foi aprovado pelo Comitê de Ética em Pesquisa do Instituto de Educação e Pesquisa do Hospital Moinhos de Vento, sob o número 2009/28.

\section{Resultados}

Dentre os 3.700 adultos e idosos elegíveis para o estudo, 309 foram considerados perdas/recusas, correspondendo a um percentual de não participantes de $8,4 \%$. A maior parte da amostra foi constituída por mulheres $(55,9 \%)$, indivíduos com companheiro(a) - (62,2\%), e com escolaridade de até oito anos de estudo (60,5\%). A idade da amostra variou de 20 a 96 anos (média $=44,1 ; \mathrm{DP}=16,0$ ). Mais detalhes referentes à descrição da amostra estão apresentados na Tabela 1.

A prevalência de depressão encontrada foi de $16,1 \%$ (IC95\%: 14,9\%; 17,4\%). Na análise bruta (Tabela 2), encontrou-se associação de depressão com sexo feminino e indivíduos separados e viúvos. Houve tendência de aumento da ocorrência de depressão em indivíduos com o avançar da idade e redução da escolaridade e nível econômico. Após análise ajustada (Tabela 2), observou-se que a depressão esteve associada ao sexo feminino, com uma probabilidade de ocorrência do desfecho 2,38 vezes maior entre as mulheres quando comparadas aos homens. Houve uma tendência de aumento da prevalência de depressão conforme o avanço da idade, com o risco mais elevado entre os adultos de 50 a 59 de idade, que apresentaram uma prevalência $75 \%$ maior, quando comparados a adultos de 20 a 29 anos. Além disso, os indicadores socioeconômicos escolaridade $\mathrm{e}$ nível econômico apresentaram uma relação inversa com a ocorrência do desfecho, isto é, quanto maior o grau de escolaridade e nível econômico, menor a probabilidade de ocorrência de depressão (valores $\mathrm{p}<0,001 \mathrm{e}$ 0,003 , respectivamente).

\section{Discussão}

Muitos sãos os estudos publicados a respeito da depressão e do uso de escalas que avaliam intensidades de sintomas, porém grande parte deles é realizada em países com características socioeconômicas diferentes do Brasil e com amostras reduzidas, o que dificulta a comparabilidade dos achados. Nosso estudo apontou para uma prevalência de depressão de $16,1 \%$, valor acima do encontrado em outro estudo que também utilizou a Escala de Depressão PósNatal de Edimburgo ${ }^{19}$. Tal fato evidencia a relevância desse transtorno para a saúde pública mundial, pois a alta prevalência desse desfecho em saúde guarda ainda 
Tabela 1 - Descrição dos adultos moradores da Restinga/Extremo Sul. Porto Alegre, Rio Grande do Sul, 2009.

Table 1 - Description of adults living in Restinga/Extremo Sul. Porto Alegre, Rio Grande do Sul, 2009.

\begin{tabular}{|c|c|c|}
\hline Variável & $\mathrm{N}$ & $\%$ \\
\hline \multicolumn{3}{|l|}{ Sexo } \\
\hline Masculino & 1496 & 44,1 \\
\hline Feminino & 1895 & 55,9 \\
\hline \multicolumn{3}{|l|}{ Idade (anos completos) } \\
\hline $20-29$ & 792 & 23,3 \\
\hline $30-39$ & 648 & 19,1 \\
\hline $40-49$ & 718 & 21,2 \\
\hline $50-59$ & 595 & 17,6 \\
\hline$\geq 60$ & 638 & 18,8 \\
\hline \multicolumn{3}{|l|}{ Situação conjugal atual } \\
\hline Com companheiro(a) & 2108 & 62,2 \\
\hline Solteiro(a) & 744 & 21,9 \\
\hline Separado(a)/viúvo(a) & 539 & 15,9 \\
\hline \multicolumn{3}{|c|}{ Escolaridade (anos completos de estudo) } \\
\hline $0-4$ & 767 & 22,7 \\
\hline $5-8$ & 1279 & 37,8 \\
\hline $9-11$ & 1053 & 31,0 \\
\hline$\geq 12$ & 288 & 8,5 \\
\hline \multicolumn{3}{|c|}{ Nível econômico em quartis (ABEP) } \\
\hline $1^{\circ}$ quartil & 1028 & 30,7 \\
\hline $2^{\circ}$ quartil & 721 & 21,5 \\
\hline $3^{\circ}$ quartil & 849 & 25,3 \\
\hline $4^{\circ}$ quartil & 753 & 22,5 \\
\hline
\end{tabular}

a Variável com maior número de informações ignoradas: $n=40$.

${ }^{a}$ Variable with the highest number of unknown information: $n=40$.

relação com fatores encontrados em contextos menos favorecidos, como pobreza, violência, baixa escolaridade, entre outros.

O ponto de corte escolhido para o diagnóstico da depressão em nosso estudo $(\geq 13$ pontos) está bem embasado na literatura e tem por intuito alcançar-se uma maior fidedignidade no diagnóstico da depressão $0^{13,14,17}$. Entretanto, mesmo tendo sido utilizada em estudos internacionais com populações de ambos os $\operatorname{sexos}^{12,13,17,22,23}$, essa escala pode estar sujeita a possíveis vieses, levando a superestimativa da prevalência de depressão, em função de ter sido originalmente criada para rastreio de depressão pós-parto, e não para diagnóstico de depressão em população geral. Além disso, o fato de a população avaliada derivar de uma região de baixa condição socioeconômica também pode ter contribuído para a alta prevalência de depressão, uma vez que, independente do instrumento utilizado, a ocorrência de depressão tem forte associação com indicadores sociais e econômicos, como baixos níveis de renda e escolaridade $7,8,18,19,26,29-31$.

Encontrou-se associação entre maiores índices de depressão em pessoas do sexo feminino, refletindo um dado bastante conhecido na epidemiologia das doenças mentais, dado esse também confirmado em outras pesquisas ${ }^{7,26}$. Possíveis explicações para essa diferença entre os sexos são 
Tabela 2 - Prevalência de depressão, análise bruta e ajustada conforme as variáveis independentes do estudo em adultos moradores da Restinga/Extremo Sul. Porto Alegre, Rio Grande do Sul, 2009.

Table 2 - Prevalence of depression, crude and adjusted analysis, according to the independent variables in the study of adults living in Restinga/Extremo Sul. Porto Alegre, Rio Grande do Sul, 2009.

\begin{tabular}{|c|c|c|c|c|c|}
\hline \multirow{2}{*}{ Variável } & \multirow{2}{*}{$\begin{array}{c}\% \\
\text { Depressão }\end{array}$} & \multicolumn{2}{|c|}{ Análise bruta } & \multicolumn{2}{|c|}{ Análise ajustada } \\
\hline & & RP (IC95\%) & Valor $\mathbf{p}$ & RP (IC95\%) & Valor $p$ \\
\hline Sexo & & & $<0,001$ & & $<0,001$ \\
\hline Masculino & 8,9 & 1,00 & & 1,00 & \\
\hline Feminino & 21,8 & $2,45(2,04 ; 2,95)$ & & $2,38(1,97 ; 2,87)$ & \\
\hline Idade (anos completos) & & & $<0,001^{*}$ & & $0,02^{*}$ \\
\hline $20-29$ & 11,2 & 1,00 & & 1,00 & \\
\hline $30-39$ & 15,0 & $1,34(1,02 ; 1,76)$ & & $1,33(1,01 ; 1,75)$ & \\
\hline $0-49$ & 18,4 & $1,65(1,28 ; 2,12)$ & & $1,56(1,20 ; 2,03)$ & \\
\hline $50-59$ & 21,1 & $1,89(1,47 ; 2,42)$ & & $1,75(1,34 ; 2,29)$ & \\
\hline$\geq 60$ & 16,3 & $1,46(1,12 ; 1,90)$ & & $1,26(0,94 ; 1,68)$ & \\
\hline Situação conjugal atual & & & $<0,001$ & & 0,13 \\
\hline Com companheiro(a) & 15,2 & 1,00 & & 1,00 & \\
\hline Solteiro(a) & 13,4 & $0,88(0,71 ; 1,09)$ & & $0,89(0,71 ; 1,10)$ & \\
\hline Separado(a)/viúvo(a) & 23,6 & $1,56(1,30 ; 1,87)$ & & $1,17(0,96 ; 1,43)$ & \\
\hline Escolaridade (anos completos de estudo) & & & $<0,001^{*}$ & & $<0,001^{*}$ \\
\hline $0-4$ & 25,2 & 1,00 & & 1,00 & \\
\hline $5-8$ & 15,9 & $0,63(0,53 ; 0,75)$ & & $0,72(0,60 ; 0,87)$ & \\
\hline $9-11$ & 12,0 & $0,48(0,39 ; 0,59)$ & & $0,57(0,45 ; 0,72)$ & \\
\hline$\geq 12$ & 8,4 & $0,33(0,22 ; 0,50)$ & & $0,41(0,27 ; 0,63)$ & \\
\hline Nível econômico em quartis (ABEP) & & & $<0,001^{*}$ & & $0,003^{*}$ \\
\hline $1^{\circ}$ quartil & 21,2 & 1,00 & & 1,00 & \\
\hline $2^{\circ}$ quartil & 16,6 & $0,78(0,64 ; 0,96)$ & & $0,89(0,73 ; 1,09)$ & \\
\hline $3^{\circ}$ quartil & 15,0 & $0,71(0,58 ; 0,86)$ & & $0,85(0,70 ; 1,04)$ & \\
\hline $4^{\circ}$ quartil & 10,3 & $0,49(0,38 ; 0,62)$ & & $0,67(0,52 ; 0,87)$ & \\
\hline
\end{tabular}

"Tendência linear do teste qui-quadrado.

"Linear trend chi-square.

questões socioculturais relacionadas com experiências adversas e atributos psicológicos associados com maior vulnerabilidade a eventos estressantes em mulheres ${ }^{27}$. Os resultados em relação às diferenças de gênero para as depressões podem ser importantes para várias ações científicas e assistenciais, inclusive com melhora da capacidade diagnóstica e maior adequação de tratamentos.

Observou-se que, quanto maior a idade, maior a ocorrência de depressão. Confirmando dados da literatura, em que adultos com depressão, alcançam maior prevalência, sendo que se mantém constante entre diferentes raças e culturas ${ }^{28}$. Estudos realizados no Brasil apontam para prevalências de depressão variando de $5,7 \%$ a $17,7 \%^{24,25}$.

Portanto, torna-se importante levar em consideração o momento de vida do paciente em que se faz esse diagnóstico. No caso de idosos, por exemplo, este quadro geralmente traz o medo de dependência durante o curso da doença e, possivelmente, até a morte. Para os mais jovens, significa ameaça a seus planos de vida, à carreira profissional, à sexualidade, à família, entre outros. Percebe-se que essas dificuldades são vivenciadas de modo individual, de 
acordo com a história de vida, recursos internos e a idade na qual se encontra o sujeito. A literatura relata que a depressão é o quarto maior agente incapacitante das funções sociais e outras atividades da vida cotidiana ${ }^{5}$.

Foi também evidenciado neste trabalho que menores níveis educacionais estão associados a maior ocorrência de depressão, em consonância com outros estudos conduzidos no Brasil ${ }^{7,31}$ Considerandose o nível econômico, observou-se uma relação inversa com o desfecho, ou seja, quanto maior o nível econômico, menor a prevalência de depressão. Outros estudos realizados também encontraram relação entre as classes mais pobres, visto que apresentaram maiores índices de depressão, comparadando-as às classes economicamente mais favorecidas ${ }^{7,9}$. Sabe-se que tanto a escolaridade quanto o nível econômico são importantes marcadores socioeconômicos. Estudos relatam que a pobreza pode ser um determinante na explicação do aumento dos índices de depressão, pois eles estão associados a condições sociais como desemprego, baixo nível de instrução, baixa qualidade de moradia e alimentação inadequada $^{27,31}$. Tais condições, exclusivas ou combinadas, podem favorecer o desenvolvimento de um estado de desesperança, que tem como efeito imediato a redução da capacidade para lidar com situações estressoras de maneira adequada, reduzindo a disposição para suportar acontecimentos adversos e frustrantes.

Não se pode desconsiderar, entretanto, a possibilidade de causalidade reversa nas associações, pois é inerente ao delineamento utilizado nas pesquisas. Por exemplo, se a depressão surgiu durante a idade estudantil do indivíduo, implicando perda de desempenho escolar e abandono precoce da escola, há possibilidade de que haja causalidade reversa entre educação e depressão, uma vez que a depressão pode ter surgido na infância ou na adolescência, prejudicando a escolaridade do doente, $\mathrm{e}$ ter então persistido durante a idade adulta.

Os estados depressivos, tanto por sua prevalência quanto pelas consequências que acarretam, têm marcante importância enquanto problema de saúde pública, reforçando que a depressão tem uma alta associação com pior funcionamento social e qualidade de vida e maior utilização de recursos de saúde em pacientes de cuidados primários. As limitações impostas, o sofrimento que acarreta e seu custo social muito grande constituem os maiores problemas e apenas parte das pessoas afetadas tem acesso ao diagnóstico e aos tratamentos adequados.

Demonstrar variações na distribuição de depressão em diferentes grupos populacionais permite um planejamento mais adequado das políticas, programas e serviços de saúde. Diferentes tipos de intervenções têm sido usados para ajudar em relação às necessidades psicológicas e sociais de sujeitos com sintomas depressivos. A atuação do psicólogo no âmbito da atenção primária à saúde representa uma dessas possibilidades, e exemplo disso seria o Núcleo de Apoio à Saúde da Família (NASF) que valoriza a ação multidisciplinar de profissionais da área da saúde, que tem como intuito ampliar a abrangência e as ações de atenção básica, melhorando a qualidade e resolutividade da atenção à saúde ${ }^{32}$. Muitas dessas intervenções são destinadas a auxiliar o indivíduo a se sentir menos desamparado e desanimado, para assumir maior responsabilidade em sua recuperação ou para aderir aos procedimentos médicos ${ }^{23,26}$.

Com isso, percebe-se desde o início do tratamento uma série de consequências psicossociais que perduram, alteram-se ou somam-se durante o quadro clínico do paciente. Essas complicações exigem sempre uma atenção especial e envolvem variados aspectos da vivência psíquica e social do sujeito e de seus familiares, em busca de uma melhor qualidade de vida. Neste sentido, é fundamental encontrar formas efetivas de avaliação da depressão, e do impacto na qualidade de vida, visto que uma avaliação correta e completa pode contribuir para propor diferentes formas de abordagens terapêuticas a esses sujeitos. 
Os resultados desta pesquisa apontam para a necessidade de que os serviços públicos ofereçam, além da intervenção usual, apoio multidisciplinar. Devido à possibilidade de tratamento desses quadros, evitando possíveis consequências graves na evolução dessa doença, sugere-se que mais estudos sejam realizados nessa área, permitindo assim atingir um maior grau de certeza quanto à verdadeira associação de fatores sociodemográficos sobre a ocorrência de depressão.

Estudos da Escala de Edimburgo (EPDS) utilizados em população masculina são incipientes, sendo realizados somente em pesquisas fora do Brasil ${ }^{22,23}$. Apesar de ter sido validado para o Brasil, em população com características sociais semelhantes às da população onde ocorreu o atual estudo, o mesmo ainda não tinha sido utilizado em amostra do sexo masculino. Contudo, o instrumento tem sido utilizado na população geral, no exterior, em estudos não somente em situações pós-natal ${ }^{12-17}$. Apesar do pequeno número de artigos de estudos brasileiros divulgados nas principais bases de dados, os resultados obtidos na pesquisa realizada contribuem para o avanço do conhecimento científico e como incentivo para a realização de novas pesquisas. Recomendam-se outros estudos, com amostra de ambos os gêneros, para verificação e confirmação da validade do instrumento para o público em geral, visto que o mesmo já está sendo utilizado com o público geral em estudos realizados em outros países.

\section{Referências}

1. World Health Organization. Equity, social determinants and public health programmes. Geneva, 2010; World Health Organization.

2. Institute of Medicine. Neurological, psychiatric and developmental disorders: meeting the challenge in the developing world. Washington, DC: National Academy Press; 2001.

3. Manual diagnóstico e estatístico de transtornos mentais: $D S M-I V .4^{\circ}$ edição. Porto Alegre, RS: Artes Médicas; 1995.

4. Organização Mundial de Saúde. Classificação internacional de doenças e problemas relacionados à saúde: CID 10. 1996-1997, 3º ed. EDUSP: São Paulo.

5. Ludermir AB, Lewis G. - Links between social class and common mental disorders in Northeast Brazil. Soc Psychiatry Psychiatr Epidemiol 2001; 36: 101-7.

6. IBGE. Síntese de indicadores sociais 2008. Disponível em: http://www.ibge.gov.br/ sinteseindicsociais2008.

7. Almeida Filho N, Lessa I, Magalhães L, Araújo MJ, Aquino E, James AS et al. Social inequality and depressive disorders in Bahia, Brazil: interactions of gender, ethnicity and social class. Soc Sci Med 2004; 59: $1339-53$.

8. Andrade L, Walters EE, Gentil V, Laurenti R. Prevalence of ICD10 Mental Disorders in a Catchment Area in the City of São Paulo, Brazil. Soc Psychiatry Psychiatr Epidemiol 2002; 37: 31625.
9. Barros, MBA, Cesar CLG, Carandina L. Desigualdades sociais na prevalência de doenças crônicas no Brasil, PNAD-2003. Ciência \& Saúde Coletiva, Rio de Janeiro, 2006; $11: 4$

10. Zavaschi, M. L. S. et al. Associação entre trauma por perda na infância e depressão na vida adulta. Rev Bras Psiquiatria 2002; 24(4): 189-95.

11. Botega NJ, Furlanetto L, Fraguas R Jr. Depressão. In Botega N J (org.). Prática Psiquiátrica no Hospital Geral: Interconsulta e Emergência. Porto Alegre: Artmed, 2006. p. 225-46.

12. Cox JL, Champman G, Murray D, Jones, P. Validation of the Edinburgh Postnatal Depression Scale (EPDS) in non-postnatal women. J Affect Dis 1996; 39(3): 185-9.

13. Nyklicek I, Scherders M J, Pop VJ. Multiple assessments of depressive symptom as index of depression in population-based samples. Psychiatry Res 2004; 128(2): 111-6.

14. Van Den Berg M, Verdijk NA, Leusink GL, Wijnands-Van Gent CJ, Romeijnders AC, Pop VJ et al. Depression after low-energy fracture in older women predicts future falls: a prospective observational study. BMC Geriatry 2011; 11: 73.

15. Lloyd-Williams, M., Shiels, C., Taylor, F., Dennis, M. Depression-an independent predictor of early death in patients with advanced cancer. J Affect Dis 2009; 113(12): 127-32. 
16. De Cock ES, Emons WH, Nefs G, Pop VJ, Pouwer F. Dimensionality and scale properties of the Edinburgh Depression Scale (EDS) in patients with type 2 diabetes mellitus: the DiaDDzoB study. BMC Psychiatry 2011; 11: 141.

17. Alexander S, Palmer C, Stone PC. Evaluation of screening instruments for depression and anxiety in breast cancer survivors. Breast Cancer Res Treat 2010, 122(2): 573-8.

18. Santos MFS, Martins FC, Pasquali L. Escala de autoavaliação de depressão pós-parto: estudo no Brasil. Rev Psiquiatr Clin 1999; 26: 90-5.

19. Da Silva VA, Moraes-Santos AR, Carvalho MS, Martins MLP, Teixeira NA. Prenatal and postnatal depression among low-income Brazilian women. Braz J Med Biol Res 1998; 31: 799-804.

20. Santos IS, Matijasevich A, Tavares BF, Barros AJD, Botelho IP, Lapolli C, Magalhães PVS, Barbosa APPN, Barros FC. Validation of Edinburgh Postanatal Depression Scale (EPDS) in a sample of mothers from the 2004 Pelotas Birth Chort Study. Cad Saúde Pública 2007; 23(x): 105-116.

21. Santos IS, Matijasevich A, Tavares BF, Cruz ACL, Riegel RE, Lopes B. Comparing validity of Edimburgh scale and SRQ20 in screening for post-partum depression. Clin Pract Epidemiol Mental Health 2007; 3: 18.

22. Edmonson OJH, Lamprini, Psychogiou L, Vlachos H, Netsi E, Ramchandani P. Depression in fathers in the postnatal period: Assessment of the Edinburgh Postnatal Depression Scale as a screening measure. J Affect Dis 2010, 125(1-3): 365-368.

23. Ramchandani P, Stein A, Evans J, O'Connor TG. ALSPAC, study team. Paternal depression in the postnatal period and child development: a prospective population study. Lancet 2005; 365(9478): 2201-5.
24. Associação Brasileira de Empresas de Pesquisa. Critério de Classificação Econômica Brasil. São Paulo; 2003. Disponível em: http://www.abep.org/codigosguias/ ABEP_CCEB_2008.pdf. [Acessado em 16 de setembro de 2010]

25. Barros AJ, Hirakata VN. Alternatives for logistical regression in cross-sectional studies: an empirical comparison of models that directly estimate the prevalence ratio. BMC Medical Res Methodol 2003; 3: 21.

26. Peluso ETP, Blay SL. Percepção da depressão pela população da cidade de São Paulo. Rev Saúde Pública 2008; 42: 1.

27. World Health Organization. Mental health: new understanding, new hope. Geneva: The world health report; 2001.

28. Simon GE. Evidence review: efficacy and effectiveness of antidepressant treatment in primary care. Gen Hosp Psychiatry 2002; 24: 213-24.

29. Cesar CLG, Carandina L, Alves MCGP, Barros MBA, Goldbaum M. (org.). Saúde e condição de vida em São Paulo. Inquérito multicêntrico de saúde no Estado de São Paulo ISA-SP. $1^{\text {a }}$ edição. São Paulo: FSP/USP; 2005.

30. Lima-Costa MF. A saúde dos adultos na Região Metropolitana de Belo Horizonte: um estudo epidemiológico de base populacional. Belo Horizonte: Nespe, Fiocruz, UFMG; 2004.

31. Mello MF, Mello AAF, Kohn R. Epidemiologia da saúde mental no Brasil. Porto Alegre: Artmed; 2007.

32. Portaria $\mathrm{N}^{\circ} 154$. Núcleo de Apoio à Saúde da Família NASF. 2008. Disponível em: http:/ / dab.saude.gov.br/ dab/nasf.php. [Acessado em 24 de novembro de 2010].

Recebido em: 10/01/2011

Versão final apresentada em: 02/03/2012

Aprovado em: 11/03/2012 